\title{
Cuidados de enfermagem na prevenção da infecção puerperal em parto cesárea: análise complementar
}

Nursing care in the prevention of puerperal infection in cesarean delivery: complementary analysis Atención de enfermería en la prevención de la infección puerperal en el parto por cesárea: análisis complementário

Ana Fátima Souza Melo de Andrade ORCID: https://orcid.org/0000-0002-7024-6175 Centro Universitário Estácio de Sergipe, Brasil E-mail: anafatimamelo@hotmail.com

Weber de Santana Teles ORCID: https://orcid.org/0000-0003-1770-8278 Centro de Hemoterapia de Sergipe, Brasil E-mail: arteecura@hotmail.com

Max Cruz da Silva

ORCID: https://orcid.org/0000-0002-6944-5986

Faculdade Pio Décimo, Brasil E-mail: maxlfi@hotmail.com

Fernanda dos Santos

ORCID: https://orcid.org/0000-0001-6443-5589 Universidade Tiradentes, Brasil E-mail: nandasantos1603@gmail.com

Gabriely Moura Gonzaga ORCID: https://orcid.org/0000-0001-5838-1991 Universidade Tiradentes, Brasil

E-mail: gabriellyenfermagem@outlook.com

Rebeca Galvão Fonseca

ORCID: https://orcid.org/0000-0002-4059-1226

Centro Universitário Estácio de Sergipe, Brasil E-mail: rebeca_galvao@yahoo.com.br

Ruth Cristini Torres

ORCID: https://orcid.org/0000-0002-8664-192X Instituto de Hematologia e Hemoterapia de Sergipe, Brasil E-mail: ruthcristini@gmail.com Alejandra Debbo

ORCID: https://orcid.org/0000-0002-7743-5921 Universidade Tiradentes, Brasil E-mail: aledebbo@hotmail.com

Marcel Vinícius Cunha Azevedo ORCID: https://orcid.org/0000-0002-5312-3333 Universidade Tiradentes, Brasil E-mail: marcelvinicius49@gmail.com

Maria Hozana Santos Silva

ORCID: https://orcid.org/0000-0001-5742-5366 Faculdade Ages de Medicina, Brasil E-mail: hosana_p@hotmail.com

Ângela Maria Melo Sá Barros ORCID: https://orcid.org/0000-0003-4087-3247 Universidade Federal do Rio de Janeiro, Brasil E-mail: angelsamelo@hotmail.com

Paulo Celso Curvelo Santos Junior ORCID: https://orcid.org/0000-0001-5834-6782 Universidade Tiradentes, Brasil E-mail: paulo.curvelo.jr@gmail.com Aline Barreto Hora

ORCID: https://orcid.org/0000-0002-3930-6475 Centro Universitário Estácio de Sergipe, Brasil E-mail: aline.barretoh@hotmail.com

Taíssa Alice Soledade Calasans ORCID: https://orcid.org/0000-0003-0460-4437 Universidade Tiradentes, Brasil E-mail: taissa.asc@gmail.com 


\title{
Resumo
}

O presente estudo tem o objetivo de avaliar o papel do Enfermeiro na prevenção da Infecção Puerperal associada à cirurgia Cesariana. Metodologia: Trata-se de uma revisão integrativa, de caráter analítico e retrospectivo, com abordagem qualitativa. A primeira etapa é composta pela delimitação do tema que foi definido como: Cuidados de Enfermagem na prevenção de Infecção Puerperal em Parto Cesariana, para responder a pergunta norteadora: Quais os Cuidados de Enfermagem no ambiente intra-hospitalar que podem prevenir a infecção puerperal no parto cesariana? Resultados: Mediante a análise dos textos completos foram avaliados na íntegra 07 artigos, estes atenderam aos critérios de inclusão expostos na metodologia desta pesquisa. Considerações Finais: Conclui-se que existem fatores de risco associados que são determinantes para a Infecção Puerperal no Parto Cesáreo e que o profissional de Enfermagem deve adotar cuidados embasados cientificamente para a aplicabilidade dos mesmos, pois, o enfermeiro é responsável por gerenciar os riscos, notificar os eventos adversos, e principalmente agir de modo a prevenir e reduzir as infecções no âmbito hospitalar.

Palavras-chave: Infecção puerperal; Cesárea; Cuidados de enfermagem; Prevenção.

\begin{abstract}
This study aims to assess the role of nurses in preventing puerperal infection associated with Cesarean surgery. Methodology: This is an integrative review, analytical and retrospective, with a qualitative approach. The first step is composed by the delimitation of the theme, which was defined as: Nursing Care in the prevention of Puerperal Infection in Cesarean Delivery, to answer the guiding question: What Nursing Care in the intra-hospital environment can prevent puerperal infection in childbirth Caesarean? Results: Through the analysis of the full texts, 07 articles were fully evaluated, which met the inclusion criteria set out in the methodology of this research. Final Considerations: It is concluded that there are associated risk factors that are determinant for Puerperal Infection in Cesarean Delivery and that the Nursing professional must adopt scientifically-based care for their applicability, as the nurse is responsible for managing the risks, notify adverse events, and mainly act in order to prevent and reduce infections in the hospital environment.
\end{abstract}

Keywords: Puerperal infection; Cesarean section; Nursing care; Prevention.

\section{Resumen}

Este estudio tiene como objetivo evaluar el papel de las enfermeras en la prevención de la infección puerperal asociada a la cesárea. Metodología: Se trata de una revisión integradora, analítica y retrospectiva, con enfoque cualitativo. El primer paso está compuesto por la delimitación del tema, que se definió como: Atención de Enfermería en la prevención de la Infección Puerperal en el Parto por Cesárea, para dar respuesta a la pregunta orientadora: ¿Qué Atención de Enfermería en el ámbito intrahospitalario puede prevenir la infección puerperal en el parto? ¿Cesariano? Resultados: A través del análisis de los textos completos, se evaluaron en su totalidad 07 artículos, los cuales cumplieron con los criterios de inclusión establecidos en la metodología de esta investigación. Consideraciones finales: Se concluye que existen factores de riesgo asociados que son determinantes para la Infección puerperal en el parto por cesárea y que el profesional de Enfermería debe adoptar cuidados con base científica para su aplicabilidad, siendo la enfermera la encargada de manejar los riesgos, notificar los eventos adversos, y actuar principalmente con el fin de prevenir y reducir las infecciones en el entorno hospitalario.

Palabras clave: Infección puerperal; Cesárea; Cuidado de enfermera; Prevención.

\section{Introdução}

As infecções relacionadas à assistência à saúde (IRAS), são acontecimentos contrários, que frequentemente tem associação ao atendimento à saúde, sendo desta forma um grande problema de saúde pública, tendo em vista o crescimento da mortalidade, e os gastos relacionados com estes procedimentos, além de atingir de forma pejorativa a seguridade dos indivíduos (Figura 1), e o sistema de gestão de qualidade dos atendimentos ofertados (ANVISA, 2021). 
Figura 1: IRAS: um problema de qualidade nos serviços de saúde.

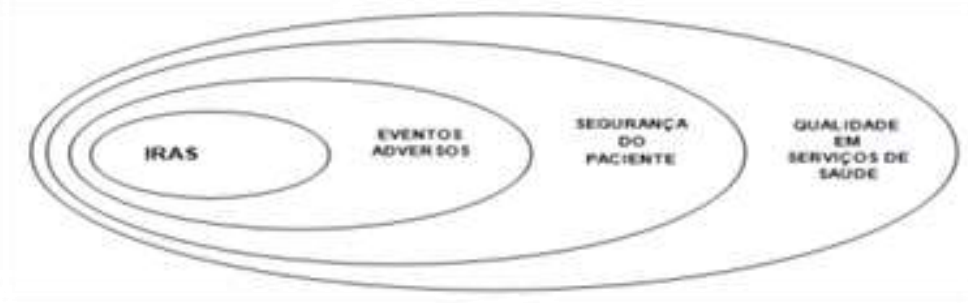

Fonte: Costa (2016).

Apesar dos indícios relacionados ao ônus da economia das IRAS, apresentar limitações, especialmente em nações que estão em crescimento, dados demonstrados nos Estados Unidos da América (EUA) e no continente Europeu, estabelecem custos em bilhões por ano. Pesquisas realizadas no território brasileiro, mostraram que a estimativa dos gastos de ocupação-dia total e médio por indivíduos com IRAS ou sem IRAS, as taxas de gastos diários com os pacientes com IRAS foi 55\% superior ao de um paciente sem IRA (ANVISA, 2021).

Conceituada como uma contaminação adquirida no hospital, o Ministério da Saúde, define como uma infecção contraída após a hospitalização do indivíduo, se manifestando durante a sua estádia ou após a alta, e que tem relação com o período em que esteve interno ou com os procedimentos operacionais do hospital (Girotto et al., 2021).

Dentre as (IRAS), as infecções de sítio cirúrgico (ISC) estão entre as mais frequentes, sendo responsáveis por $38 \%$ de todas as infecções hospitalares, ocasionando assim um aumento significativo no número de internações, custos e morbimortalidade. Ainda sobre as ISC, destaca-se a Infecção Puerperal,que têm comodefinição qualquer processo infeccioso inflamatório do trato genital que ocorra no período do puerpério, podendo desenvolver: Febre maior que $38^{\circ} \mathrm{C}$, com manifestação após 24 horas do parto e duração mínima de 2 dias; esta éresponsável por 10\% das mortes maternas ao ano principalmente nos países subdesenvolvidos (Cunha et al., 2018).

Se tratando das cirurgias obstétricas, o parto cirúrgico é indicado quando surge algum tipo de risco para a mãe e o bebê,o procedimento é realizado no intuito de intervir e salvar suas vidas. No entanto, a realização de cesáreas no Brasil atingiu a taxa de $82,3 \%$ no setor privado e de 33,25\% no Sistema Único de Saúde (SUS), dados divergentes aos $15 \%$ recomendado pela Organização Mundial da Saúde (OMS), estudos apontam que as taxas de cesáreas no Brasil aumentam de forma significativa pois estão associadas a escolaridade materna e são mais indicadas para as classes favorecidas sendo provavelmente sem indicação clínica (Carvalho et al., 2014).

A taxa alta de incidência de parto cesariano tem como consequência o aumento da morbimortalidade materna e neonatal, tendo riscos aumentados em até 1,5 vezes a mais em relação ao parto vaginal, caracterizando-se como um problema. Na América Latina, as cesáreas sem indicação são responsáveis pela ocorrência, a cada ano, de 100 mortes maternas e 40.000 casos de doença respiratória neonatal (Reis et al., 2017).

Estudos de incidência das ISCs pós-cesarianas, mostrados em pesquisas internacionais e brasileiras, destacam que mais de $80 \%$ das mulheres que desenvolveram a infecção tiveram o início dos sintomas após a alta hospitalar, em até 15 dias após o parto, evidenciando a importância do acompanhamento da puérpera no ambiente do domicílio e na Atenção Básica, seja na consulta de puerpério ou no momento daretirada de pontos cirúrgicos (Cunha et al., 2014).

As ISCs pós cesáreas são as mais comuns dentro das IRAS, sendo $60 \%$ possível de prevenção a partir de medidas de controle e boas práticas. Estas ações são executadas em três momentos dentro do âmbito hospitalar no: Pré-parto com a realização do banho pré-operatório e tricotomia 2 horas antes do parto; Intra-operatório através da administração da antibioticoprofilaxia, manutenção das portas e janelas da sala operatória fechadas durante o ato curúrgico, restrição do número de pessoas na sala 
operatória; e no Pós-parto, com atenção para o pós-operatório imediato, realização do checklist de cirurgia segura, manutenção correta do curativo,entre outras medidas de prevenção (Brasil, 2017).

Buscando priorizar os procedimentos de minimização dos riscos, as ações da ANVISA (Agência Nacional de Vigilância Sanitária) no que diz respeito ao nível de perigos e ameaças do serviço, serão efetuados nos estabelecimentos assistenciais de saúde em todas as suas complexidades, práticas de segurança do paciente, e em resposta a essas ações deverão ser apresentados um plano de atendimento as não conformidades, seguidamente da monitorização quanto ao atendimento das solicitações, atendendo prazo estabelecido pela ANVISA. (ANVISA, 2021).

Figura 2: Modelo teórico da gestão do risco sanitário baseado no monitoramento da implantação de Práticas de Segurança do Paciente.

\begin{tabular}{|c|c|c|c|c|c|}
\hline \multicolumn{3}{|c|}{ AVALIACAOO DO RISCO } & \multicolumn{3}{|c|}{ GERENCIAMENTO DO RISCO } \\
\hline $\begin{array}{l}\text { Identificacalo do } \\
\text { nisco pelo servico de } \\
\text { saùde }\end{array}$ & $\begin{array}{c}\text { Análise pela Vigilância } \\
\text { Sanitána }\end{array}$ & $\begin{array}{c}\text { Avaliaçắo pela Vigilância } \\
\text { Sanitária }\end{array}$ & $\begin{array}{l}\text { Açäo pela Vigiáncia } \\
\text { Sanitária }\end{array}$ & $\begin{array}{l}\text { Monitoramento pela } \\
\text { Vigiläncia Sanitária }\end{array}$ & $\begin{array}{l}\text { Comunicaçåo pela } \\
\text { Vigilancia Sanitaria }\end{array}$ \\
\hline \multirow{3}{*}{$\begin{array}{l}\text { Envio da Avaliaç̄o } \\
\text { Anual das Práticas } \\
\text { de Segurança do } \\
\text { Paciente }\end{array}$} & $\begin{array}{l}\text { Sorteio de servicos de } \\
\text { saude para inspecido } \\
\text { in loco dos dados } \\
\text { enviados }\end{array}$ & $\begin{array}{l}\text { ALTA CONFORMIDADE } \\
\text { Conformidade em 67\%. } \\
100 \% \text { de indicadores, } \\
\text { inclusive os indicadores } 1 \\
\text { (Núcleo) e } 18 \text { (Notificaça) }\end{array}$ & & & \multirow{4}{*}{$\begin{array}{c}\text { Publicar anualmente } \\
\text { lista dos serviços de } \\
\text { saûde classificados } \\
\text { como "Alta } \\
\text { Conformidade" as } \\
\text { Práticas de } \\
\text { Segurança do } \\
\text { Paciente }\end{array}$} \\
\hline & \multirow{2}{*}{$\begin{array}{l}\text { Revisāo dos dados } \\
\text { enwiados utilizando o } \\
\text { "Instrutivo para } \\
\text { Análise do Formulário } \\
\text { de Avaliaça das } \\
\text { Práticas de Seguranca } \\
\text { do Paciente" }\end{array}$} & $\begin{array}{l}\text { MEDIA CONFORMIDADE } \\
\text { Conformidade em } 34 \% \text { - } \\
66 \% \text { dos indicadores }\end{array}$ & $\begin{array}{l}\text { Solicitar adequaçäo às } \\
\text { Práticas de Segurança do } \\
\text { Paciente com prazo } \\
\text { definido }\end{array}$ & \multirow{3}{*}{$\begin{array}{l}\text { Monitorar cumprimento } \\
\text { das metas no prazo } \\
\text { estabelecido }\end{array}$} & \\
\hline & & \multirow{2}{*}{$\begin{array}{l}\text { BAIXA CONFORMIDADE } \\
\text { Conformutede em 0\%\%-33\% } \\
\text { dos indicadores }\end{array}$} & $\begin{array}{l}\text { Determinar adequação } \\
\text { às Práticas de Segurança } \\
\text { do Paciente com prazo } \\
\text { definido }\end{array}$ & & \\
\hline $\begin{array}{ll}\text { NAO envio da } \\
\text { Avaliaçäo Anual de } \\
\text { Práticas } & \text { de } \\
\text { Segurança } & \text { do } \\
\text { Paciente } & \\
\end{array}$ & & & $\begin{array}{l}\text { Determinar o envio da } \\
\text { Avaliaçăo das Práticas de } \\
\text { Segurança do Paciente } \\
\text { com prazo definido }\end{array}$ & & \\
\hline Fe & $\begin{array}{r}\text { Indicadores } \\
\text { ura: \% de servicos de } \\
\text { i Proce } \\
\text { + Práticas de Seguran }\end{array}$ & $\begin{array}{l}\text { mplementaç̧o do Plano Ir } \\
\text { de conformes quanto a pres } \\
\% \text { de servicos de saude ca } \\
\text { do Paciente em geral. } \% \text { de }\end{array}$ & $\begin{array}{l}\text { egrado no municipio, esta } \\
\text { aca de Nucleos, Planos e Pr } \\
\text { formes quanto á adesão ao } \\
\text { ervicos de saude classificad, }\end{array}$ & $\begin{array}{l}\text { do/DF e nacional: } \\
\text { otocolos de Seguranca d } \\
\text { s Protocolos } \\
\text { os como alla contormidax }\end{array}$ & aciente \\
\hline
\end{tabular}

Fonte: Plano Integrado para a Gestão Sanitária da Segurança do Paciente em Serviços de Saúde 2021 - 2025. Anvisa (2021).

A responsabilidade da prevenção e controle das IRAS dentro das instituições de saúde, cabe a todos os colaboradores da equipe, seja ela multidisciplinar ou interdisciplinar. Dentro deste contexto é importante ressaltar a atuação da equipe de Enfermagem, já que a mesma representa $60 \%$ do quadro de profissionais de saúde e é a categoria que executa a assistência diretamente ao paciente (Monteiro et al., 2015).

Diante desta vertente, a atuação da equipe de enfermagem se torna necessária para a prevenção das IRAS. Estudos apontam que a lavagem das mãos; uso de Equipamento de Proteção Individual (EPI'S); Educação Permanente a cada 1 mês com a equipe, são simples medidas preventivas cotidianas da equipe de Enfermagem que irão garantir uma assistência com foco na qualidade, minimizando os riscos e consequentemente os possíveis eventos adversos decorrentes ao cuidado prestado ao paciente (Brasil, 2017).

Para melhor entendimento da temática, a pesquisa traz como relevância o destaque das principais medidas preventivas para Infecção Puerperal por via de parto cesariana, uma vez que o puerpério é um período de risco e crítico tornando os cuidados de enfermagem indispensáveis e essenciais, contribuindo para a prevenção e redução das taxas de infecção puerperal. Desta 
forma a pesquisa tem como objetivo principal avaliar o papel do Enfermeiro para a prevenção da Infecção Puerperal associada à cirurgia cesariana.

\section{Metodologia}

Trata-se de uma revisão integrativa, de caráter analítico e retrospectivo, com abordagem qualitativa. A escolha da revisão integrativa foi selecionada por ter a finalidade de resumir resultados de pesquisas para um determinado tema escolhido, desta forma os leitores podem contemplar um estudo mais específico e aprofundado dobre o assunto (Soares, 2014).

Para que a revisão integrativa seja desenvolvida é necessário percorrer e respeitar as seguintes etapas: estabelecimento de objetivos da revisão integrativa; estabelecimento de critérios de inclusão e exclusão de artigos (seleção da amostra); definição das informações a serem extraídas dos artigos selecionados; análise dos resultados; discussão e apresentação dos resultados (Souza et al., 2010).

A primeira etapa é composta pela delimitação do tema que foi definido como: Cuidados de Enfermagem na prevenção de Infecção Puerperal em Parto Cesariana. Afim de para responder a seguinte questão norteadora: Quais os Cuidados de Enfermagem no ambiente intra-hospitalar que podem prevenir a infecção puerperal no parto cesariana?

Para considerar a segunda etapa, foi realizada uma busca dos dados no dia 21 de outubro de 2019, nas respectivas bases de dados: Literatura Latino Americana e do Caribe em Ciências da Saúde (LILACS), Medical Literature Analysis and Retrieval System Online (MEDLINE) e a coleção Scientific Eetronic Library online (SciELO) e Base de Dados de Enfermagem (BDENF). As palavras utilizadas foram estabelecidasa partir de consulta nos Descritores em Ciências da Saúde (DeCS). A estratégia de busca utilizou os termos em inglês : ("Puerperal Infection"; "Nursing Care”; "Cesarean”; "Prevention”) e português ("Infecção Puerperal", “Cuidados de Enfermagem"; "Cesárea”; e "Prevenção"), sendo utilizadoo operador boleano AND.

Logo após esta etapa, foi realizada uma seleção manual executada em seis etapas: 1) Rejeição de artigos duplicados; 2) Inspeção de todos os títulos dos artigos incluídos; 3) Avaliação dos resumos dos artigos no qual os títulos foram selecionados; 4) Análise dos artigos completos com leitura minunciosa; 5) Organização manual da identificação dos artigos incluídos após a leitura na íntegra; 6) Extração e análise dos dados.

Seguindo esse método, da primeira a quarta etapa, a verificação dos artigos encontrados através da estratégia de busca por seleção, ocorreu de forma independente, com a finalidade de garantir maior exatidãopara explanar os estudos que possuiam potencial de relevância para inclusão, utilizando os critérios de elegibilidade predeterminados. Esta seleção foi realizada por dois revisores (F.S; G.M.G), e as discrepâncias entre os revisores foram analisadas, julgadas e resolvidas por um terceiro revisor (R. G. F).

Para refinamento da pesquisa, foram estabelecidos os critérios de inclusão: apenas artigos publicados entre 2010 a 2019 que pesquisaram a assistência de Enfermagem à puérpera na prevenção da infecção do sítio cirúrgico em parto cesariana. Foram excluídos deste estudo os artigos que não estavam disponíveis na íntegra, boletins epidemiológicos, teses e recortes de revistas, jornais, carta ao editor, congressos resumos, revisões sistemáticas e metanálises, estudos que abordavam exclusivamente de cuidados farmacológicos voltados à equipe médica.

Na quinta etapa em diante, dois revisores (F.S; G.M.G) realizaram a seleção manual da identificação dos artigos após a leitura completa dos mesmos. A partir disso, foi realizada a elaboração de um quadro com as seguintes informações: Título; Autor; Periódico/Ano, Metodologia, País, Principais resultados; Principais cuidados de Enfermagem; e Fatores que favorecem a Infecção Puerperal.

A amostra inicial conteve 160 artigos, sendo: 112 (LILACS); 35 (Scielo); 02 (PUBMED); 01 (MEDLINE); 03 (BDENF). As bases de dados, juntamente com a estratégia de busca, número de artigos encontrados e as informações estão expostas no Fluxograma 1. 
Após a coleta de dados, realizou-se a seleção dos primeiros artigos conforme a pergunta norteadora e os critérios de inclusão previamente definidos, foi realizado um refinamento destes por ano de publicação de 2009 à 2019 onde foram excluídos 64 artigos duplicados. A partir disso foi realizada a leitura e análise por título e resumo, que resultou na exclusão de 52 artigos.

Diante das análises dos títulos e resumos dos artigos, observou-se que 24 artigos eram potencialmente relevantes para a pesquisa, destes foram excluídos 11 devido o texto está incompleto e indisponíveis nas bases de dados. Após restaram 13 artigos que foram lidos na íntegra, destes 06 foram excluídos por: ano de publicação < 2009; Idioma em Italiano; e assuntos sobre: Parto Humanizado; e Mães portadoras de HIV, revisões sistemáticas, metanálises e revisão integrativa.

Mediante a análise dos textos completos foram avaliados na íntegra 07 artigos, estes atenderam aos critérios de inclusão expostos na metodologia desta pesquisa. Vale ressaltar a importância na seleção destes artigos finais, visto que todos respondam à questão norteadora delimitada.

Os 07 artigos preencheram os critérios de inclusão e compuseram a amostra final (Petter et al., 2013, Mascarello et al., 2018, Araujo et al., 2019, Junior et al., 2009, Cunha et al., 2018, Cavalcante et al., 2013, \& Benincasa, 2012). O fluxograma ilustrado abaixo detalha o processo de seleção e o número de artigos excluídos em cada etapa da revisão integrativa.

Fluxograma 1: Processo de Seleção dos Estudos.

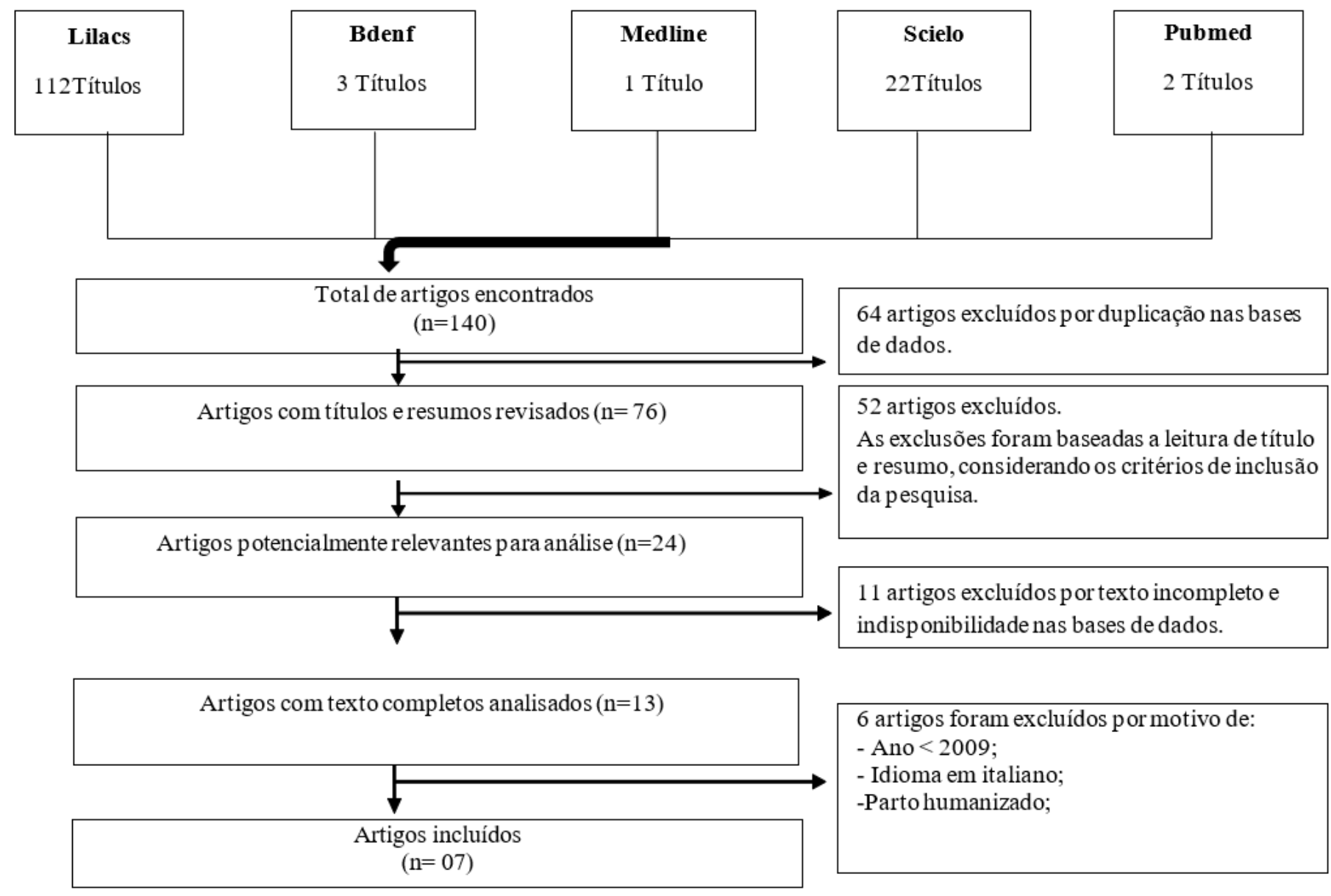

Fonte: Autores (2019). 


\section{Resultados e Discussão}

O aumento das cesáreas tem sido apontada ao longo dos anos na literatura, entre 2009-2013, depois entre 2018-2019. A partir do ano de 2009 já era perceptível o aumento das taxas de cesarianas no Brasil e no mundo, Júnior (8), aborda em seu artigo sobre a superindicação das cesáreas tanto no Brasil como internacionalmente, ainda traz um dado importante em que está indicação tem predominância nas classes socioeconômicas mais altas, e que com esse aumento, ocasiona complicações maternas e neonatais.

Os artigos que retratam sobre a infecção puerperal aparecem de forma sucinta na literatura dentro do período analisado. Nos anos de 2009 à 2013, com um total de 57,2\% (n=4), sendo que em 2013 foram publicados 2 artigos; e nos anos de 20182019, com um total de 42,9\% (n=3) sendo que em 2018 foram 2 publicações. O menor ano de publicação foi em 2009 14,3\% ( $\mathrm{n}=01)$ dos estudos incluídos, e o mais atual foi em 2019 14,3\% (n=01).

\subsection{Caracterização Metodológica}

No que diz respeito a caracterização metodológica, $87 \%(\mathrm{n}=06)$ apresentam abordagem quantitativa, entre estes, $50 \%$ $(n=3)$ representam estudos transversais, e 3 a outra metade (50\%) são estudos de coorte. Dentre os 07 artigos selecionados apenas $14,3 \%(\mathrm{n}=01)$ a abordagem é qualitativa e o tipo de estudo é coorte. As temáticas que tiveram destaque perante a análise dos estudos foram: "Fatores de risco para o desenvolvimento da infecção puerperal; complicações puerperais; Caracterização da Infecção Puerperal e suas taxas".

Exatamente $87 \%(\mathrm{n}=06)$ desses artigos foram publicados no Brasil e apenas 14,3\% ( $\mathrm{n}=01)$ na Costa Rica, isto demonstra que existe mais estudos no Brasil, devido a taxa alta de parto cesariana ao longo dos anos quando comparado a outros países. Mascarello (10), enfatiza no estudo que a taxa de partos cesarianas no Brasil era de cerca de 15\%, subindo para 38\% em 2001 e para 48,8\% em 2008, representando 35\% dos partos do Sistema Único de Saúde (SUS) e 80\% dos partos do setor privado. Nenhum outro país (China, EUA, e Alemanha) superou as taxas de cesarianas do Brasil.

No que tange a respeito dos objetivos do estudo, os artigos tiveram como foco identificar os fatores de risco que favorecem as infecções puerperais em parto cesariana e identificar as medidas de prevenção e controle mais utilizadas nas etapas da cirurgia cesariana. Sendo assim, o foco visualizado foi direcionado ao entendimento a respeito da prevenção infecciosa. As tabelas 1 e 2, de síntese a seguir mostram as informações extraídas dos 07 artigos selecionados. 
Research, Society and Development, v. 10, n. 13, e459101321435, 2021

(CC BY 4.0) | ISSN 2525-3409 | DOI: http://dx.doi.org/10.33448/rsd-v10i13.21435

Tabela 1: Características primárias dos estudos incluídos na revisão integrativa.

\begin{tabular}{|c|c|c|c|c|c|}
\hline $\mathbf{N}^{\circ}$ & Título & Autor (es) & $\begin{array}{l}\text { Periódico/An } \\
\text { o }\end{array}$ & Metodologia do Estudo & País \\
\hline 1 & $\begin{array}{l}\text { Fatores relacionados a infecções de sítio } \\
\text { cirúrgico após } \\
\text { procedimentos obstétricos. }\end{array}$ & $\begin{array}{l}\text { Catarina Escosteguy Petter; Túlio Cícero } \\
\text { Franco Farret; Juliane de Souza Scherer; } \\
\text { Vicente Sperb Antonello. }\end{array}$ & 2013 & $\begin{array}{l}\text { Estudo transversal, retrospectivo e } \\
\text { descritivo, quantitativo. }\end{array}$ & Brasil \\
\hline 2 & $\begin{array}{l}\text { Complicações puerperais precoces e } \\
\text { tardias } \\
\text { associadas à via de parto em uma coorte } \\
\text { no Brasil }\end{array}$ & $\begin{array}{l}\text { Keila Cristina Mascarello; Alicia } \\
\text { Matijasevich; Iná da Silva dos Santos; } \\
\text { Mariângela Freitas Silveira. }\end{array}$ & 2018 & $\begin{array}{l}\text { Estudo Coorte, prospectivo, e } \\
\text { quantitativo. }\end{array}$ & Brasil \\
\hline 3 & $\begin{array}{lll}\text { Ocorrência de infecções de } & \text { sítio } \\
\text { cirúrgico pós-cesárea em } & \text { uma } \\
\text { maternidade pública } & & \end{array}$ & $\begin{array}{l}\text { Andréa Bárbara Santana de Araújo; Janmilli } \\
\text { da Costa Dantas; Francisca Marta de Lima } \\
\text { Costa Souza; Bárbara Coeli Oliveira da } \\
\text { Silva; Wenysson Noleto dos Santos; Débora } \\
\text { Thaís de Aguiar Sena. }\end{array}$ & 2019 & $\begin{array}{l}\text { Estudo Transversal, retrospectivo, } \\
\text { quantitativo. }\end{array}$ & Costa Rica \\
\hline 4 & $\begin{array}{l}\text { Associação entre via de parto e } \\
\text { complicações maternas em hospital } \\
\text { público da Grande São Paulo, Brasil }\end{array}$ & $\begin{array}{l}\text { Luís Carlos Machado Junior; Christian Eric } \\
\text { Sevrin; Emerson de Oliveira; Heráclito } \\
\text { Barbosa de Carvalho; Jorge Washington } \\
\text { Zamboni; José Carlos de Araújo; } \\
\text { MarilandeMarcolin; Paulo Caruso; Pedro } \\
\text { Ferreira Awada; Ricardo Zanetti Giunta; } \\
\text { Wirley Munhoz; Mauro Sancovski; Sérgio } \\
\text { Peixoto. }\end{array}$ & 2009 & $\begin{array}{l}\text { Estudo de coorte, retrospectivo, } \\
\text { qualitativo. }\end{array}$ & Brasil \\
\hline 5 & $\begin{array}{l}\text { Identificação da infecção de sítio } \\
\text { cirúrgico pós-cesariana: consulta de } \\
\text { enfermagem }\end{array}$ & $\begin{array}{l}\text { Marcia Regina Cunha;Maria } \\
\text { Clara Padoveze; Célia Regina Maganha } \\
\text { e Melo;Lucia Yasukolzumi Nichiata. }\end{array}$ & 2018 & $\begin{array}{l}\text { Estudo quantitativo, exploratório, } \\
\text { descritivo, transversal e } \\
\text { retrospectivo. }\end{array}$ & Brasil \\
\hline 6 & $\begin{array}{l}\text { Caracterização das infecções puerperais } \\
\text { em uma maternidade pública municipal } \\
\text { de Teresina em } 2013\end{array}$ & $\begin{array}{l}\text { Milena France Alves Cavalcante; Verbenia } \\
\text { Cipriano Feitosa; Filipe Augusto de Freitas } \\
\text { Soares; Daniela Costa Araújo. }\end{array}$ & 2013 & $\begin{array}{l}\text { Estudo retrospectivo, de caráter } \\
\text { descritivo e exploratório com } \\
\text { abordagem quantitativa. }\end{array}$ & Brasil \\
\hline 7 & $\begin{array}{l}\text { Taxas de infecção relacionadas a partos } \\
\text { cesáreos e normais no Hospital de } \\
\text { Clínicas de Porto Alegre }\end{array}$ & $\begin{array}{l}\text { Bianca Chassot Benincasa; Caroline } \\
\text { Walker; Christine Cioba; Cibele Corbellini } \\
\text { da Silva Rosa; Daiana Eltz } \\
\text { Martins;Enderson Dias; Mariza Kluck. }\end{array}$ & 2012 & $\begin{array}{l}\text { Estudo de coorte retrospectivo, de } \\
\text { caráter observacional. }\end{array}$ & Brasil \\
\hline
\end{tabular}

Fonte: Autores (2019). 


\subsection{Fatores de Risco Encontrados}

De acordo com os estudos analisados, $57,14 \%(\mathrm{n}=4)$, apontam a primiparidade um fator de risco para o desenvolvimento da Infecção Puerperal. Tal hipótese pode ser justificada pela indicação do procedimento cirúrgico, escolha induzida com o objetivo de promover mais conforto à mulher, poupá-la da dor do parto normal e pelo fato de insinuar que a primípara, no âmbito anatômico não está bem preparada para um parto normal. Os médicos obstetras indicam com o intuito de protegê-las, uma vez que são consideradas susceptíveis a traumas perineais e outras disfunções do assoalho pélvico, portanto não existem estudos que associem a esses riscos nas nulíparas (Cavalcante, 2013). Muitas vezes as mulheres são submetidas a partos cirúrgicos sem nenhuma indicação médica clara, por conveniência devido à solicitação materna ou recomendação médica sem justificativa clínica. Além disso, as taxas de cesárea acima desse limite não têm demonstrado benefício adicional para a mãe ou o bebê (Mascarello et al., 2018).

Outro fator de risco relevante foi a obesidade, representado em $42,85 \%(n=3)$ dos estudos quatro em número real, que está bem documentada na literatura como fator de risco para infecção pós-operatória. Mulheres com IMC maior do que 30 são mais propensas a desenvolver infecção de ferida operatória do que mulheres com IMC normal (Magann et al., 2011). O índice de massa corpórea, indicando sobrepeso ou obesidade é fator contribuinte, devido à circulação tecidual ineficaz, ao maior acúmulo de seromas, à hematomas e à presença de tecido subcutâneo maior que dois centímetros, promovendo um risco maior de deiscências e consequentemente ISC (Cunha et al., 2018).

O tempo de cirurgia também foi um grande fator observado, 28,57\% $(n=2)$ dos estudos apontam que um grande tempo cirúrgico aumenta a chance do desenvolvimento dessa complicação. Isso se deve a uma maior exposição da parturiente à microrganismos existentes no ambiente e com isso deve se adotar medidas de preventivas. Uma medida simples que deve ser adotada é o controle de circulantes dentro da sala cirúrgica.

A assistência ao parto no Brasil deve ser entendida a partir de inúmeros motivos específicos quanto a atenção à saúde reprodutiva, dentre estes fatores estão inclusas grandes evidências de esterilização e partos cesáreas, baixa excelência de atenção obstétrica, práticas estas que refletem da má realização do pré-natal, da assistência desumana ao parto e da procura de gestantes em trabalho de parto sobre leitos hospitalares (Frello, 2011).

\subsection{Cuidados de Enfermagem}

De acordo com os dados expostos na tabela 2, os cuidados de Enfermagem encontrados de forma semelhante nos estudos foram: Administração da antibioticoprofilaxia de 30 a 60 minutos antes da cirurgia; Degermação com clorexidina a 2\%; Tricotomia e o banho pré-operatório e aplicação da SAE (Sistematização da Assistência de Enfermagem); 28,57\%(n=2) dos estudos não apresentaram os cuidados de maneira clara.

Quanto ao preparo cirúrgico, segundo a literatura, o banho deve ser realizado previamente ao procedimento e envolver todo o corpo. A tricotomia por sua vez, deve ser realizada fora da sala de cirurgia, preferencialmente com tricotomizadores elétricos, imediatamente antes do procedimento, não pressionando a pele com muita força e, após o término do procedimento a área deve ser higienizada com água morna e sabonete antisséptico (Brasil, 2017).

A respeito da administração da antibioticoprofilaxia, 42,58\% ( $n=3)$ dos estudos enfatizam como cuidado essencial, devendo o enfermeiro o papel desse preparo e administração de maneira correta do fármaco. A administração antibiótica profilática 60 minutos antes da incisão da pele, comparada com o uso do antibiótico após o clampeamento do cordão umbilical, foi associada à redução de $40 \%$ na endometrite pós-parto e de 30\% na ISC. Nota-se que em 42,85\% (n=3) da amostra, não foram apresentados cuidados de Enfermagem para a prevenção (Owens et al., 2009).

Quanto as etapas do Processo de Enfermagem, a menos realizada é a etapa do diagnóstico de enfermagem. Os motivos para tal dificuldade na implementação completa da SAE podem estar relacionados a uma insuficiente base de conhecimentos 
acerca das taxonomias diagnósticas, incapacidade de estabelecer diagnósticos pautados nas necessidades dos pacientes e a falta de exercícios práticos. A SAE promove um cuidado de enfermagem de modo mais efetivo, seja nas ações preventivas, de controle e redução de danos associados às infecções puerperais (Cavalcante, 2013).

A consulta de puerpério deve obedecer aos requisitos do processo de enfermagem que constitui, em sua primeira etapa, a coleta de dados ou o histórico de enfermagem, caracterizada pelas informações da pessoa, família, comunidade e o processo saúde-doença. A etapa seguinte trata do diagnóstico de enfermagem que tem como objetivo subsidiar a tomada de decisão, de maneira a alcançar os resultados esperados (Cunha, 2018).

\section{Considerações Finais}

Conclui-se que existem fatores de risco associados que são determinantes para a Infecção Puerperal no Parto Cesáreo e que o profissional de Enfermagem deve adotar cuidados embasados cientificamente para a aplicabilidade dos mesmos.

Esta pesquisa possibilitou o aprofundamento da temática sobre os Cuidados de Enfermagem na prevenção da Infecção Puerperal trazendo como benefícios, uma análise de estudos originais na qual remete a importância do profissional Enfermeiro na prevenção desse tipo de infecção e apresenta relevância para a categoria profissional, contribuindo com o fortalecimento do processo de trabalho em todas as fases e principalmente na gestão de riscos. O Enfermeiro é responsável por gerenciar os riscos, notificar os eventos adversos, e principalmente agir de modo a prevenir e reduzir as infecções no âmbito hospitalar.

Considerando um tema pertinente e a necessidade de estudos voltados para o mesmo, o presente estudo torna-se benéfico para a contribuição de conteúdo que estará presente em base de dados podendo ser replicado em novas pesquisas e demonstra a direta atuação do profissional de enfermagem frente à temática, uma vez que atualmente há um baixo número de artigos originais e completos acerca do tema. Além disso, foi encontrada a superficialidade do papel do enfermeiro nos artigos, a maioria não tratando diretamente do envolvimento e atuação do profissional de enfermagem acerca do tema.

\section{Referências}

Anvisa. (2021). Plano Integrado para a Gestão Sanitária da Segurança do Paciente em Serviços de Saúde 2021 - 2025.

Anvisa. (2021). Programa nacional de prevenção e controle de infecções relacionadas à assistência à saúde (PNPCIRAS) 2021 a 2025. Gerência de Vigilância e Monitoramento em Serviços de Saúde - GVIMS. Brasília.

Anvisa. (2021). Orientações para preenchimento da avaliação das práticas de segurança do paciente - 2021. Gerência de Vigilância e Monitoramento em Serviços de Saúde. Brasília.

Brasil. (2017). Manual de Medidas de Prevenção e Critérios Diagnósticos de Infecções Puerperais em Parto Vaginal e Cirurgia Cesariana. Manual, (8a ed.).

Brasil. (2017). Manual Medidas de Prevenção de Infecção Relacionada à Saúde. Manual, (4a ed.),

Carvalho, I., Souza, N., \& Medeiros, A. (2014). Risk factors for wound infection after cesarean section: an integrative review. Revista de Pesquisa: Cuidado é Fundamental Online. 6(2) 812-820.

Cavalcante, M. F. A., Feitosa, V. C., Soares, F. A. F., \& Araujo, D. C. (2013). Caracterização das infecções puerperais em uma maternidade pública municipal de Teresina em 2013. Rev Epidemiologia e Controle de Infecção. 2(1).

Costa, M. M. M. (2016). Efeitos de um ciclo de melhoria da qualidade nacional aplicado à estruturação das ações de prevenção das infecções relacionadas à assistência à saúde em hospitais brasileiros. Dissertação (Mestrado) - Curso de Mestrado Profissional Gestão da Qualidade em Serviços da Saúde, Centro de Ciências da Saúde, Universidade Federal do Rio Grande do Norte, Natal (RN).

Cunha, R. M., Padoveze, M. C., Celia, R., Luda, Y., \& Izumi, N. (2018). Identificação da infecção de sítio cirúrgico pós-cesariana: Consulta de enfermagem. Rev Brasileira de Enfermagem. 71(2). 1478-1486.

Frello, A. T., Carraro, T. E., \& Bernardi, M. C. (2011). Cuidado e conforto no parto: estudos na enfermagem brasileira. Revista Baiana de Enfermagem, 25(2), $150-159$.

Junior, L. C. M., Sevrin, C. E., Oliveira, E., Carvalho, H. B., Zamboni, J. W., \& Araujo, J. C. (2009). Associação entre via de parto e complicações maternas em hospital público da Grande São Paulo, Brasil. Cad. Saúde Pública, 25. 
Research, Society and Development, v. 10, n. 13, e459101321435, 2021

(CC BY 4.0) | ISSN 2525-3409 | DOI: http://dx.doi.org/10.33448/rsd-v10i13.21435

Magann, E. F., Doberty, D. A., Chauban, S. P. (2011). Pregnancy, obesity, gestationalweightgain, andparity as predictorsofpeeripartumcomplications. Arch Gynecol Obstet. 284, 827-36.

Mascarello, K. C., Matijasevich, A., Santos, I. S., \& Silveira, M. S. (2018). Complicações puerperais precoces e tardias associadas à via de parto em uma coorte no Brasil. Rev Bras. Epidemiologia, v.21.

Monteiro, T. S., \& Pedroza, R. M. (2015). Infecção hospitalar: visão dos profissionais da equipe de enfermagem. Rev. Epidem Control Infect. 10(2).

Owens, S. M., Brozanski, B. S., \& Meyn, L. A. (2009). Antimicrobial prophylaxis for cesarean delivery before skinin cision. Obstet Gynecol. 114, 573-9.

Reis, T. L. R et al. (2017). Autonomia feminina no processo de parto e nascimento: revisão integrativa da literatura. Rev Gaúcha Enferm. 38 (1), e64677.

Sena, L. M. (2017). Violência obstétrica no Brasil e o ciberativismo de mulheres mães: relato de duas experiências. Rev.Comunic. saúde educação. 21(60), 20920 .

Silva, T. P. R. et al. (2020). Fatores associados ao parto normal e cesárea em maternidades públicas e privadas: estudo transversal. Rev. Bras. Enferm. 73, (Suppl 4), e20180996.

Soares, C. B., Hoga, L. A. K., Peduzzi, M., Sangaleti, C., Yonekura, T., \& Silva, D. R. A. D. (2014). Revisão integrativa: conceitos e métodos utilizados na enfermagem. Rev. Esc. Enfermagem USP. 10(1).

Souza, M. T., Silva, M. D., \& Carvalho, R. (2010). Revisão integrativa: o que é e como fazer. Einstein. 8(1), 102-6. 